\title{
Aufsatz Grundlagenfach
}

\section{Prof. Dr. Andreas Piekenbrock \\ Der Weihnachtsmann, der Osterhase und die (Rechts-)Wissenschaft}

DOI 10.1515/jura-2015-0073

\section{Einleitung}

Die Rechtswissenschaft ist eine sprachgebundene Wissenschaft ${ }^{1}$. Der Rechtswissenschaftler bedient sich nicht nur selbst der Sprache, um über das Recht nachzudenken und seine Erkenntnisse mit der Gemeinschaft zu teilen, zu kommunizieren. Vielmehr ist auch der Gegenstand der Rechtswissenschaft, das Recht, immer sprachgebunden. Soweit es sich um positives, um gesetztes Recht handelt, bedient sich der Gesetzgeber zur Verkündung in der Regel der Sprache $^{2}$; graphische Darstellungen ${ }^{3}$ oder mathematische Formeln ${ }^{4}$ sind dagegen die Ausnahme. Bei der Verwendung sprachlicher Zeichen ist seit Babylon ${ }^{5}$ die zum Teil hochpolitische Auswahl zu treffen, welche Sprache verwendet wird $^{6}$. Da sich der Gesetzgeber im Rechtsstaat

1 Vgl. etwa Rüthers/Fischer/Birk Rechtstheorie, 7. Aufl. 2013, Rn 150. 2 Die Norm mit der Sprache gleichzusetzen, ist dagegen eine un zulässige Reduktion, ein ontologischer Fehler.

3 Vgl. dazu etwa die Straßenverkehrszeichen in den Anlagen zur StVO sowie die Darstellung von Hoheitszeichen in der Anordnung über die deutschen Flaggen vom 13. 11. 1996, BGBl I, 1729 und von Münzen in der Bekanntmachung über die Ausprägung von deutschen Euro-Gedenkmünzen im Nennwert von 2 Euro (Gedenkmünze »Niedersachsen«) vom 28. 2. 2014, BGBl I, 253.

4 Vgl. etwa § 32a I EStG.

5 Vgl. 1. Mos. (Gen.) 11, 7-9.

6 So erscheinen im spanischen »Boletín Oficial del Estado « Anhänge in baskischer, gallicischer, katalanischer und valencianischer Sprache. Vgl. www.boe.es/diario_boe. In Finnland werden alle Gesetze in der »Finlands Författningssamling « auch auf Schwedisch verkündet. In Belgien werden Gesetze im zweisprachigen »Moniteur Belge«bzw. »Belgisch Staatsblad« verkündet. Dort werden föderale Rechtstexte gelegentlich auch auf Deutsch publiziert, weil es nach der Verfassung eine deutschsprachige Gemeinschaft (Art. 2) und ein deutsches Sprachgebiet (Art. 4 I) gibt. Vgl. www.ejustice.just.fgov.be/cgi/welco me.pl. In Italien wurden die Verfassung und wichtige Gesetzbücher

Andreas Piekenbrock: Der Autor ist Inhaber des Lehrstuhls für Bürgerliches Recht und Insolvenzrecht unter besonderer Berücksichtigung ihrer europäischen Bezüge an der Ruprecht-Karls-Universität Heidelberg. möglichst verständlich ausdrücken muss ${ }^{7}$, muss er die Sprache(n) auswählen, die von den Gesetzesadressaten ${ }^{8}$ gesprochen wird bzw. werden'. So hat der Rat der EWG in seiner ersten Verordnung alle vier Amtssprachen der sechs Gründungsstaaten zu Amts- und Arbeitssprachen erhoben ${ }^{10}$; heute sind es $24^{11}$. In Deutschland wird bei völkerrechtlichen Dokumenten, die keinen verbindlichen deut-

wie das Zivilgesetzbuch, der »codice civile«, für den amtlichen Gebrauch in Südtirol ins Deutsche übersetzt. Die »Gazzetta Ufficiale« erscheint aber nur auf Italienisch. In der Schweiz ist Rätoromanisch nach der Bundesverfassung von 1999 zwar Landessprache (Art. 4), aber keine allgemeine Amtssprache des Bundes (Art. 70 I). Bundesgesetze werden daher nicht auf Rätoromanisch veröffentlicht. Russisch ist in Estland, Lettland und Litauen nicht zweite Amtssprache, obwohl es jeweils große russischsprachige Minderheiten gibt. Diese Staaten haben auch die Europäische Charta der Regional- oder Minderheitensprachen vom 5. 11. 1992, BGBl 1998 II, 1314 nicht unterzeichnet.

7 Zum verfassungsrechtlichen Grundsatz der Normenklarheit vgl. etwa BVerfGE 108, 1, 20; 108, 53, 75; 110, 33, 54. Der BFH wollte aus der Verletzung dieses Grundsatzes die Verfassungswidrigkeit der Mindestbesteuerung nach $\S \S 2$ III, 10 d EStG i.d.F. von Art. $1 \mathrm{Nr} 1,18$ des Steuerentlastungsgesetzes 1999/2000/2002 vom 24. 3. 1999, BGBl I, 402 herleiten. Vgl. BFHE 214, 430, 441ff. Dem ist das BVerfG allerdings nicht gefolgt, sondern hat die Vorlage nach Art. 100 I GG als unzulässig angesehen. Vgl. BVerfGE 127, 335, 355.

8 Zur Frage, wer als Adressat anzusehen ist, vgl. jüngst Towfigh JA 2015, 81, 85f., der auf »Juristen in ihrer Rolle als (erste) Vermittler des Rechts« abstellen will. Dagegen hat der BFH bei Steuergesetzen den Steuerpflichtigen als Normadressaten angesehen. Vgl. BFHE 214, 430, 440.

9 So ist das österreichische »Reichsgesetzblatt für die im Reichsrath vertretenen Königreiche und Länder« von 1870 bis 1918 auch auf Italienisch, Kroatisch, Polnisch, Rumänisch, Ruthenisch, Slowenisch und Tschechisch erschienen. Vgl. http://alex.onb.ac.at/rbg_nicht deutsch.htm.

10 Vgl. Art. 1 der Verordnung Nr 1 vom 15. 4. 1958 zur Regelung der Sprachenfrage für die Europäische Wirtschaftsgemeinschaft, $\mathrm{ABl} \mathrm{Nr}$ 17, 385: Deutsch, Französisch, Italienisch, Niederländisch.

11 Vgl. Art. 1 der Verordnung Nr 1 (Fn. 10) i.d.F. von Art. 1 I lit p i.V.m. Abschnitt $18 \mathrm{Nr} 2$ des Anhangs zur Verordnung (EU) Nr 517/ 2013 des Rates vom 13. 5. 2013, ABl Nr L 158, 1, 71: Bulgarisch, Dänisch, Deutsch, Englisch, Estnisch, Finnisch, Französisch, Griechisch, Irisch, Italienisch, Kroatisch, Lettisch, Litauisch, Maltesisch, Niederländisch, Polnisch, Portugiesisch, Rumänisch, Schwedisch, Slowakisch, Slowenisch, Spanisch, Tschechisch und Ungarisch. Tür- 
schen Text enthalten, stets eine unverbindliche deutsche Übersetzung veröffentlicht ${ }^{12}$.

Dass sich die Auswahl der Sprache am Sprachgebrauch der Adressaten orientiert, ist aber nicht gleichbedeutend mit der Identität zwischen der jeweiligen Alltagsund der Rechtssprache. So kann ein und dasselbe Wort verschieden verwendet werden: In der Alltagssprache leihen wir uns ein Blatt Papier, zehn Euro oder ein Fahrrad; in der Rechtssprache kann dagegen beim Blatt Papier eine Schenkung (§ 516 BGB), bei den zehn Euro ein Darlehen ( $\$ 488$ BGB) und beim Fahrrad eine Miete ( 535 BGB) vorliegen. Auch innerhalb der Rechtssprache kann die Bedeutung ein und desselben Wortes in verschiedenen Kontexten divergieren. Ein klassisches Beispiel ist das Wort »Eigentum« im BGB und Art. 14 I GG.

Hier von einer Divergenz zwischen dem »natürlichen Wortsinn $\aleph^{13}$ und der Rechtssprache zu sprechen, wäre allerdings falsch, weil die Sprache nicht Teil der Natur, sondern der vom Menschen erschaffenen Kultur ist. Daher basiert jede Kommunikation mittels Zeichen auf einer Konvention unter den Teilnehmern, so dass es einen »natürlichen Wortsinn « oder ein »natürliches Sprachempfinden « ${ }^{14}$ gar nicht gibt. Vielmehr gibt es nur einen üblichen, der allgemeinen Konvention entsprechenden Wortsinn. Wenn der Gesetzgeber Wörter in diesem üblichen Wortsinn verwendet, erleichtert er das Verständnis des Gesetzes. Gleichwohl kann er Wörter auch in einem ihm eigenen, möglicherweise unkonventionellen Sinn verwenden, wenn er den Sprachgebrauch gesetzlich mit verständlichen Worten definiert. So heißt es im Kontext des Gemeinnützigkeitsrechts in $\S 52$ II $1 \mathrm{Nr} 21$ der Abgabenordnung (AO): »Schach gilt als Sport«. Allerdings darf der Gesetzgeber dabei nicht so weit gehen wie Peter Bichsel in seiner kleinen Geschichte "Ein Tisch ist ein Tisch ${ }^{15}$, in der ein alter Mann so viele Wörter umdefinierte, dass er mit niemandem mehr reden konnte ${ }^{16}$. Das wäre mit dem verfassungsrechtlichen Gebot der Normenklarheit nicht zu vereinbaren.

kisch gehört nicht dazu, obwohl es auf Zypern nach der Verfassung von 1960 offiziell die zweite Amtssprache der Republik ist (Art. 3 I).

12 Vgl. etwa die EMRK vom 4. 11. 1950, BGBl 1952 II, 686.

13 So etwa BVerfG NJW 2001, 1848, 1850; BVerfGE 131, 316 Rn 77; BGHSt 25, 347, 351; BGHSt 28, 129, 132; BGH NJW-RR 1991, 1061, 1062 »Königl. Bayerische Weisse«; BGH NStZ 2012, 35 Rn 67.

14 So etwa BGHSt 22, 235, 236.

15 Bichsel, Ein Tisch ist ein Tisch, Frankfurt am Main: Suhrkamp, 1995; www.deutschunddeutlich.de/contentLD/GD/GT67cTischistTisch.pdf.

16 Das Experiment endete mit dem Satz: »Am Mann blieb der alte Fuß lange im Bild läuten, um neun stellte das Fotoalbum, der Fuß fror auf und blätterte sich aus dem Schrank, damit er nicht an den Morgen schaute.«

\section{Der Weihnachtsmann, der Osterhase und die Reichsschokoladenverordnung}

\section{Die Verbreitung einer kleinen amüsanten Geschichte}

Ein besonders bekanntes Beispiel für den gesetzlich definierten Sprachgebrauch sind Legaldefinitionen, die im BGB weit verstreut zu finden sind ${ }^{17}$. Dazu schreibt Bernd Rüthers:

Ein schönes Beispiel dafür, daß Legaldefinitionen nichts anderes als vom Gesetzgeber festgelegte Sprachgebrauchsvereinbarungen sind, ist die Bestimmung einer »Reichsschokoladenverordnung « der dreißiger Jahre, in der angeordnet wurde: "Weihnachtsmänner im Sinne dieser Regelung sind auch Osterhasen.« Der Sache nach war das die Ausdehnung des Geltungsbereiches einer Gesetzesvorschrift auf die Osterhasenproduktion ${ }^{18}$.

Dieselbe Geschichte, deren Regelungskontext zunächst offenbleibt, erzählt Christian Förster:

Hübsch auch die Reichsschokoladenverordnung: „Weihnachtsmänner im Sinne dieser Verordnung sind auch Osterhasen. « ${ }^{19}$

Er sieht darin allerdings ein Beispiel für eine Fiktion und nicht für eine Legaldefinition. Diese Deutung ist überzeugender, weil die Fiktion das als ungleich Erkannte bewusst gleichbehandelt und damit eine verdeckte Verweisungsnorm darstellt ${ }^{20}$. Allerdings bleiben auch Legaldefinitionen nicht immer innerhalb des üblichen Wortsinns. So würde wohl niemand Eier, Kälbchen und Mieterträge gemeinhin als Früchte bezeichnen (§99 I, III BGB) ${ }^{21}$. Erst recht ist ein massives Gebäude, das nach § 95 I BGB nicht wesentlicher Bestandteil des Grundstücks ist, auf dem es steht, im üblichen Wortsinn keine bewegliche Sache, wohl aber für den Juristen ${ }^{22}$.

17 Vgl. etwa §§ 90, 90a S 1, 91, 92, 93, 97, 99, 100, 121 I, 122 II, 166 II, 183, 184 I, 194 I, 273 I, 274 I, 305 I 1, 310 III, 312b, 312c, 312i I, 383 III, 421 S 1428 S 1478 I, 491 I BGB.

18 So erstmals Rüthers Rechtstheorie, 2. Aufl. 2005, Rn 131a; vgl. entsprechend Rüthers/Fischer/Birk (Fn. 1) Rn 131a.

19 Förster Allgemeiner Teil des BGB, 2. Aufl. 2011, Rn 125.

20 Vgl. nur Larenz Methodenlehre der Rechtswissenschaft, 6. Aufl. 1991, 262 und Rüthers/Fischer/Birk (Fn. 1), Rn 132a, beide unter Verweis auf $\S 119$ II BGB. Das prominenteste Beispiel für eine gesetzliche Fiktion ist das Erbrecht des Nasciturus in $\S 1923$ II BGB.

21 Vgl. nur MünchKomm-BGB/Stresemann § 99 Rn 2, 6; BGH NJWRR 2009, 1522 Rn 23.

22 Vgl. nur BGHZ 23, 57, 59; NJW-RR 2006, 1160 Rn 7. 
In anderen Disziplinen führt die Sprachkunst der Juristen zu Erstaunen. So schreibt der Wirtschaftswissenschaftler Konrad Scorl zu unserer kleinen Geschichte:

Wie sollte ein Studierender der Wirtschaftswissenschaft oder der Rechte nachvollziehen können, dass für den Juristen der Weihnachtsmann auch ein Osterhase sein kann? ${ }^{5}$ [Fn. 5: So aber die Reichsschokoladenverordnung der dreißiger Jahre: „Weihnachtsmänner im Sinne dieser Regelung sind auch die Osterhasen«; zitiert nach Rüthers (Fn. 4) Rn. 13a; ... ${ }^{23}$.

Welchen Zweck der Verordnungsgeber mit der Gleichsetzung der als ungleich erkannten Schokoladenfiguren verfolgt haben soll, erfahren wir schließlich aus einem Artikel im Spiegel aus dem Jahr 1966. Dort wird kein geringerer als der langjährige Sprecher des Vorstandes der Deutschen Bank, Hermann Josef Abs, der nach dem ZWeiten Weltkrieg für die Bundesrepublik das Londoner Schuldenabkommen ausgehandelt und unterzeichnet hat ${ }^{24}$, mit den Worten zitiert:

Im Kriege gab es die Verordnung, daß Weihnachtsmänner nicht hergestellt werden dürften, um Kakao zu sparen. Und zu Ostern wurde bestimmt, daß im Sinne dieser Verordnung Osterhasen wie Weihnachtsmänner zu betrachten seien ${ }^{25}$.

Daraus wird immerhin deutlich, dass es um die Erweiterung eines Verbotstatbestandes durch einen sprachlichen Kunstgriff des Verordnungsgebers gegangen sein soll.

\section{Die Suche nach der Quelle der Erkenntnis}

\section{a) Empirischer Befund}

Gibt man sich als (Rechts-)Wissenschaftler mit der Weitergabe dieser so amüsanten wie lehrreichen Geschichte vom Hörensagen nicht zufrieden, muss man nach der Quelle suchen, in der sie zu finden sein soll: der »Reichsschokoladenverordnung der dreißiger Jahre. Selbst wenn man das Suchfeld auf die Zeit von 1919 bis 1945 ausweitet, findet sich in den Teilen I und II des Reichsgesetzblatts jedoch kein Hinweis auf Weihnachtsmänner oder Osterhasen. Geht man bei der Suche chronologisch vor, erfährt

23 Scor Recht im Dialog, Gedächtnisschrift für Rainer Wörlen, 2013, 669, 670. Der Nachweis ist allerdings nicht ganz korrekt, weil das Wort »die« hinzugefügt und die Randnummer fehlerhaft übertragen worden.

24 Vgl. das Abkommen über deutsche Auslandsschulden vom 27. 2. 1953, BGBl II, 333, 366.

25 Der Spiegel Nr 18/1966 vom 25. 4. 1966, S. 56, www.spiegel.de/ spiegel/print/d-46406995.html. man zunächst, dass die Verordnung über die Herstellung von Süßigkeiten und Schokolade aus dem ersten Weltkrieg $^{26}$, die die Verarbeitung von Zucker auf die Hälfte beschränkt hatte ${ }^{27}$, im Juli 1921 aufgehoben worden is $\mathrm{t}^{28}$. In der Kriegsverordnung stößt man aber nur auf »Christbaum-« und »Osterzuckersachen ${ }^{29}$. Als sich die Wirtschaftslage 1922 durch die ungelöste Kriegsschuldenfrage verschlechterte, wurde die Verwendung inländischen $\mathrm{Zu}$ ckers zur Herstellung von Schokolade verboten ${ }^{30}$; ab 1923 konnte sie wieder genehmigt werden ${ }^{31}$; 1924 fiel auch der Genehmigungsvorbehalt weg ${ }^{32}$. Von Weihnachtsmännern und Osterhasen ist aber auch hier nichts zu sehen. Dasselbe gilt für die lauterkeitsrechtlichen Regelungen über die zulässigen Größen von Schokoladentafeln ${ }^{33}$.

Im eigentlichen Suchfeld, den dreißiger Jahren, springt zunächst die »Verordnung über Kakao und Kakaoerzeugnisse« von $1933^{34}$ ins Auge. Wie die heutige »Kakaoverordnung $\aleph^{35}$ regelte sie aber nur die verschiedenen Arten von Schokolade und ihre Qualität. Im selben Jahr begann der Aufbau des »Reichsnährstandes«, der die Erzeugung landwirtschaftlicher Erzeugnisse regeln durfte ${ }^{36}$. In diesem Zusammenhang wurde 1935 der Zusammenschluss der deutschen Süßwarenwirtschaft verordnet ${ }^{37}$. Im August 1939 wurde zur "Sicherung der Versorgung der Bevölkerung und Wehrmacht" die öffentliche Bewirtschaftung landwirtschaftlicher Erzeugnisse eingeführt ${ }^{38}$

26 Bekanntmachung (Verordnung) vom 16. 12. 1915, RGBl S. 821. 27 Vgl. § 1 I der Verordnung vom 16. 12. 1915 i.d.F. der Verordnung vom 28. 2. 1916, RGBl S. 125. Später oblag die Versorgung mit Zucker nach $\S 1$ der Verordnung vom 14. 9. 1916, RGBl S. 1032 der Reichszuckerstelle. Daher wurde § 1 der Verordnung vom 16. 12. 1915 durch $\S 36$ der Verordnung vom 14. 9. 1916 aufgehoben.

28 So die Verordnung vom 1. 7. 1921, RGBl I, 803.

29 Vgl. die Definition von Süßigkeiten in § 3 II der Verordnung vom 16. 12. 1915.

30 So § 1 I 1 der Verordnung über Lebensmittel vom 8. 9. 1922, RGBl I, 725. Zum Hintergrund dieser Verordnung vgl. Torp Konsum und Politik in der Weimarer Republik, 2011, 253.

31 Vgl. § 9 I Nr 3 der Verordnung über Zucker vom 9. 10. 1923, RGBl I, 936

32 Die Regelung wurde durch die Verordnung vom 31. 3. 1924, RGBl I, 395 außer Kraft gesetzt.

33 Vgl. §§ 1, 2 der Verordnung über den Handel mit Tafelschokolade vom 11. 12. 1925, RGBl I, 467.

34 Verordnung vom 15. 7. 1933, RGBl I, 504.

35 Verordnung vom 15. 12. 2003, BGBl I, 2738

36 Vgl. § 1 des Gesetzes vom 13. 9. 1933, RGBl I, 626. Zur Auflösung vgl. § 1 des Gesetztes vom 21. 1. 1948. GVBI des Wirtschaftsrates des Vereinigten Wirtschaftsgebietes (WiGBL) S. 21.

37 Verordnung vom 7. 6. 1935, RGBl I, 742, ersetzt durch die Verordnung vom 7. 1. 1943, RGBI I, 22, aufgehoben durch $\S 8$ des Gesetzes vom 21. 1. 1948

38 So § 1 der Verordnung vom 27. 8. 1939, RGBl I, 1521. 
und unmittelbar nach Kriegsbeginn für Rohkakao und Süßwaren konkretisiert ${ }^{39}$. Sonstige sachdienliche Hinweise sind dem Reichsgesetzblatt nicht zu entnehmen.

Dieser empirische Befund spricht dafür, dass es die fraglichen »Reichsschokoladenverordnung « gar nicht gibt. Allerdings ist es eine wissenschaftsmethodische Herausforderung nachzuweisen, dass es etwas nicht gibt. Viel leichter wäre es gewesen, das Gegenteil, also die Existenz der besagten Verordnung nachzuweisen, indem einer der Autoren die Fundstelle in einem amtlichen Verkündungsorgan zitiert hätte. Gleichwohl kann der Beweis, dass es die Verordnung nie gegeben hat, gelingen, weil es nur um den negativen konkreten Beweis in einem abgeschlossenen Kontext geht. Wenn sich in allen seinerzeit publizierten Verkündungsorganen kein Hinweis auf Weihnachtsmänner oder Osterhasen findet, ist der empirische Beweis geführt, dass es sich bei unserer Geschichte selbst um einen Mythos, eine Fiktion handelt.

\section{b) Theoretischer Befund}

Allerdings ist diese Beweisführung fehleranfällig, weil mit Blick auf den zeitlichen Abstand ein möglicherweise einschlägiges Verkündungsorgan leicht übersehen werden kann. Dann war das Suchfeld zu klein. Darüber hinaus ist niemand davor gefeit, auch innerhalb des ausgewählten Suchfeldes etwas zu übersehen. Daher soll die empirische Beweisführung durch eine theoretische ergänzt werden. Dazu wird der systematischen Frage nachgegangen, ob die Existenz der fraglichen Regelung zu Weihnachtsmännern und Osterhasen innerhalb der Gesamtrechtsordnung plausibel ist. Dagegen spricht entscheidend, dass die 1935 gegründete Wirtschaftliche Vereinigung der deutschen Süßwarenwirtschaft im Zuge der öffentlichen Bewirtschaftung von Rohkakao und Süßwaren Ende September 1939 angeordnet hat: "Saison-, Phantasie- und figürliche Artikel aller Art dürfen nicht mehr hergestellt werden. $\aleph^{40} \mathrm{Da}$ Schokoladenweihnachtsmänner und -osterhasen als »figürliche Saisonartikel« offensichtlich von diesem allgemeinen

39 Vgl. im Einzelnen die Verordnung vom 7. 9. 1939, RGBl I, 1735. Die Definition der Süßwaren, zu denen selbstverständlich auch Kakaoerzeugnisse zählten, ergab sich aus § 1 II der Verordnung vom 7. 6.1935.

40 So Nr I.2. der Anordnung Nr 96 »Neue Herstellungsvorschriften für Kakaoerzeugnisse, Zuckerwaren und Dauerbackwaren « vom 29. 9. 1939, RNVbl (Verkündungsblatt des Reichsnährstandes) S. 729, auch abgedruckt bei Holthöfer/Juckenack, Lebensmittelgesetz, 2. Aufl., Band 2, 1941, 398. Vgl. wortgleich Nr I.2. der Anordnung Nr 122 vom 5. 6. 1941 für den Bereich der Landesbauernschaften DanzigWestpreußen und Wartheland, RNVbl S. 212.
Verbot erfasst waren, ist es nicht plausibel, dass es daneben noch ein spezielles Verbot von Schokoladenweihnachtsmännern gab, das zur Vermeidung einer Regelungslücke durch eine sprachliche Fiktion auf Osterhasen hätte erstreckt werden müssen.

\section{Der Weihnachtsmann, der Osterhase und der OGH für die Britische Zone}

Bevor wir unsere Geschichte endgültig als Fiktion entlarven können, müssen wir aber noch einer zweiten Spur nachgehen. So erzählt Dirk Fabricius eine ganz ähnliche Geschichte für die Nachkriegszeit, in der es aber um die Erweiterung eines Erlaubnistatbestandes durch einen sprachlichen Kunstgriff des Gesetzgebers gehen soll. Er schreibt:

Etwas Wesentliches von der Jurisprudenz erhascht man mit dem
Satz Weihnachtsmann im Sinne des Gesetzes ist auch der Oster-
hase. Keine Satire, sondern ein Urteilsspruch, der den Kindern
im Nachkriegsdeutschland Schokoladenosterhasen verschaffte.
Das Gesetz der Besatzungsregierung hatte die Produktion von
Schokoladenweihnachtsmännern gestattet, für ein neues Gesetz
bis Ostern war es zu kurz. Das Gericht - der oberste Gerichtshof
der Britischen Zone - musste entscheiden, ob die Produktion
von Schokoladenosterhasen unter dieses Gesetz falle bzw. es
unterzubringen sei. Denkt man sich hypothetisch Schokoladen-
panzer oder Schokoladenreichsadler, so wird deutlich, dass der
Urteilsspruch eine Ähnlichkeit behauptet, dass man jedenfalls
Schokoladenosterhasen als Schokoladenweihnachtsmännern
ähnlich ansehen könne. Mithin sei es im Sinne des Gesetzes, es
auch Ostern gelten zu lassen ${ }^{41}$.

Leider führt auch dieser Hinweis nicht weiter. Für diese Spur ist der empirische Befund besonders gut zu erheben, weil sich die Sammlung der Erkenntnisse des Obersten Gerichtshofes (OGH) für die Britische Zone ${ }^{42}$ vollständig in der Bibliothek des Bundesgerichtshofs befindet, der am 1. Oktober 1950 die Funktionsnachfolge des OGH angetre-

41 Fabricius Kriminalwissenschaften: Grundlagen und Grundfragen: Teilband 1: Darwins angetretenes Erbe - Evolutionsbiologie auch für Nicht-Biologen, 2011, 118.

42 Zur Gründung des OGH für die Britische Zone in Köln vgl. die Verordnung der Militärregierung Nr 98 vom 1. 9. 1947, ABl der Militärregierung Deutschland - Britisches Kontrollgebiet, S. $572=$ VOBl für die Britische Zone, S. 154 und dazu die Durchführungsverordnung des Zentral-Justizamtes für die Britische Zone vom 17. 11. 1947, VOBl für die Britische Zone, S. 149. 
ten hat ${ }^{43}$. Die Sammlung umfasst acht Bände mit Erkenntnissen in Zivilsachen ${ }^{44}$ und elf Bände mit Erkenntnissen in Strafsachen ${ }^{45}$ sowie ein einbändiges, inzwischen von Werner Schubert herausgegebenes und damit leicht zugängliches Nachschlagewerk ${ }^{46}$. Auch dort ist von Weihnachtsmännern und Osterhasen keine Spur. Dasselbe gilt für die einschlägigen Verkündigungsorgane, in denen das »Gesetz der Besatzungregierung « zu finden sein müsste ${ }^{47}$, und das zeitgenössische Fachschrifttum ${ }^{48}$.

$43 \mathrm{Vgl}$. Art. 8 Nr 88 des Gesetzes zur Wiederherstellung der Rechtseinheit auf dem Gebiete der Gerichtsverfassung, der bürgerlichen Rechtspflege, des Strafverfahrens und des Kostenrechts vom 12. 9. $1950, \mathrm{BGBl} \mathrm{I}, 455,509$.

44 Diese Bände sind fortlaufend nummeriert: Band 1: Entscheidungen des Zivilsenats zu den Aktenzeichen ZS 1/48-166/48; Band 2: Entscheidungen des I. Zivilsenats zu den Aktenzeichen I ZS 16/48254/48; Band 3: Entscheidungen des I. Zivilsenats zu den Aktenzeichen I ZS 1/49-66/50; Band 4: Entscheidungen des II. Zivilsenats zu den Aktenzeichen II ZS 1/48-96/48; Band 5: Entscheidungen des II. Zivilsenats zu den Aktenzeichen II ZS 3/49-117/49; Band 5: Entscheidungen des II. Zivilsenats zu den Aktenzeichen II ZS 3/49-117/49; Band 6: Entscheidungen des II. Zivilsenats zu den Aktenzeichen II ZS 120/49-238/50; Band 7: Entscheidungen des II. Zivilsenats in Landwirtschaftssachen $\mathrm{zu}$ den Aktenzeichen II Blw 2/49-56/50; Band 8: Entscheidungen des I. und II. Zivilsenats in AR-, ZA und ZBsachen 1948-1950.

45 Dies sind im Einzelnen: ein Band mit Entscheidungen aus dem Jahr 1948; zwei Bände mit den Entscheidungen zu den Aktenzeichen StS 1/48-171/48; drei Bände mit Entscheidungen aus dem Jahr 1949; zwei Bände mit den Entscheidungen zu den Aktenzeichen StS 1/49532/49; zwei Bände mit Entscheidungen des 1. und des 2. Strafsenats aus dem Jahr 1950; ein Band mit Entscheidungen zu den Aktenzeichen 1 StS 2/50-123/50 bzw. 2 StS 3/50-101/50. Hier wurden alle (kriegs-)wirtschaftsrechtlichen Fälle überprüft.

46 Vgl. Schubert (Hrsg.), Oberster Gerichtshof für die Britische Zone (1948-1950), Nachschlagewerk Strafsachen - Nachschlagewerk Zivilsachen, Präjudizienbuch der Zivilsenate, 2010.

47 Eingesehen wurden das ABI des Kontrollrats in Deutschland, Nr. 1 vom 29. 10. 1945 bis Nr. 19 vom 31. 8. 1948, das GVBI des Wirtschaftsrates des Vereinigten Wirtschaftsgebietes, Nr. 1 vom 21. 8. 1947 bis Nr. 34 vom 6. 9. 1949, das ABI der Militärregierung Deutschland Britisches Kontrollgebiet, Nr. 4 bis Nr. 28 und das VOBI. für die Britische Zone, Nr. 1 vom 23. 4.1947 bis Nr. 65 vom 13. 12. 1949.

$48 \mathrm{Vgl}$. Holthöfer/Juckenack Lebensmittelgesetz, 3. Aufl., Band II, 1953, $181 \mathrm{ff}$. (Kakao und Kakaoerzeugnisse).

\section{Ergebnisse}

Damit bleibt als erstes Ergebnis unserer Untersuchung ein ernüchternder Befund. Mit der Rechtsquelle, in der sich unsere Geschichte vom Osterhasen, der wie ein Weihnachtsmann zu behandeln ist, finden soll, verhält es sich wie mit den beiden Protagonisten selbst: Keiner hat sie je gesehen. Bis sie doch jemand sieht, dürfen und müssen wir daher annehmen, dass es sie nicht gibt. Sie alle sind Fiktionen, so wie der Inhalt der Geschichte selbst. Das hindert aber weder unsere Geschichte noch den Weihnachtsmann noch den Osterhasen, sich als Kulturgüter im kollektiven Gedächtnis einer Zunft (der Juristen) oder sogar eines beachtlichen Teils der Menschheit zu etablieren. Auch wenn sich unsere Geschichte nicht so abgespielt hat, wie behauptet wird, ist sie gut ausgedacht und lehrreich. Denn sie verdeutlicht den möglichen Umgang mit der Sprache im Recht und zeigt, was dem Gesetzgeber auf diesem Gebiet alles zugetraut wird. Anders als bei Loriots gemeiner Steinlaus (Petrophaga lorioti) ${ }^{49}$ und Christian Morgensterns Nasobēm ${ }^{50}$ bleibt der Schöpfer der Geschichte allerdings unbekannt.

Weit über ihr eigentliches Thema hinaus kann man aus unserer Geschichte lernen, dass die ungeprüfte Übernahme fremder Quellen vom Hörensagen untunlich ist. Hier gilt: Nec licet Iovi nec licet bovi. Vielmehr sind Originalquellen zu konsultieren und zu zitieren. Das gilt für heutige und frühere sowie für in- und ausländische Gesetzestexte, für Gerichtsentscheidungen und für rechtswissenschaftliche Abhandlungen. Nur wenn die Quelle nicht greifbar oder sprachlich nicht zugänglich ist, ist das Sekundärzitat vom Hörensagen zulässig, muss aber als solches offengelegt werden. Das kann durch Zusätze wie "zitiert nach « oder "deutsche Übersetzung von" geschehen. Jede andere Vorgehensweise schafft kein Wissen, sondern dient nur der Verbreitung des Irrtums. Das ist nicht die Aufgabe der (Rechts-)Wissenschaft.

49 Pschyrembel Klinisches Wörterbuch, 265. Aufl. 2014, 2020 f. 50 Morgenstern Galgenlieder, 1998, 279. 